\title{
$\beta$-Amyloid oligomers in aging and Alzheimer's disease
}

\author{
Kathleen R. Zahs ${ }^{1,2}$ and Karen H. Ashe $1,2,3,4 *$ \\ ${ }^{1}$ N. Bud Grossman Center for Memory Research and Care, University of Minnesota, Minneapolis, MN, USA \\ ${ }^{2}$ Department of Neurology, University of Minnesota, Minneapolis, MN, USA \\ ${ }^{3}$ Department of Neuroscience, University of Minnesota, Minneapolis, MN, USA \\ ${ }^{4}$ Geriatric Research Education Clinical Center, VA Medical Center, Minneapolis, MN, USA
}

\section{Edited by:}

Orly Lazarov, The University of Illinois

at Chicago, USA

\section{Reviewed by:}

Karl Herrup, Case Western University, USA

Junming Wang, University of

Mississippi Medical Center, USA

*Correspondence:

Karen H. Ashe, N. Bud Grossman Center for Memory Research and Care, University of Minnesota, Room 5-144, Wallin Medical Biosciences Building, 2101 6th Street SE, Minneapolis, MN 55455, USA e-mail: hsiao005@umn.edu

Alzheimer's disease (AD) is a fatal neurodegenerative disorder, and the most common cause of dementia in the elderly. The cause of $A D$ is not known, but genetic evidence strongly supports the hypothesis that pathological aggregation of the $\beta$-amyloid protein $(A \beta)$ triggers the disease process. AD has a long preclinical phase, lasting a decade or more. It is during this preclinical phase, before the irreversible neuron loss that characterizes the dementia phase of the disease, that therapies are most likely to be effective. If we are to block $A D$ during the preclinical phase, we must identify the $A \beta$ species that are present before there are overt symptoms and that are associated with downstream markers of pathology. A specific soluble $A \beta$ assembly, the putative dodecamer " $A \beta^{*} 56$," is present in the brains and cerebrospinal fluid of cognitively intact individuals and correlates with markers of synaptic dysfunction and neuronal injury. This assembly also correlates with memory dysfunction in multiple lines of transgenic mice that model the preclinical phase of $A D$. We suggest that $A \beta^{*} 56$ has a critical role during the earliest phase of $A D$ and might serve as a molecular trigger of the disease.

\section{Keywords: $\beta$-amyloid, oligomer, Alzheimer's disease, $A \beta * 56$, preclinical Alzheimer's disease}

\section{INTRODUCTION}

Alzheimer's disease (AD) is the most common cause of dementia in the elderly. Age is the greatest risk factor for $\mathrm{AD}$; an estimated $13 \%$ of Americans over age 65 suffer from $\mathrm{AD}$, while that number increases to $45 \%$ for those aged 85 and older (Alzheimer's Association, 2012). The disease is defined by characteristic lesions in the brain - amyloid plaques composed of aggregated $\beta$-amyloid protein $(A \beta)$, neurofibrillary tangles composed of hyperphosphorylated tau protein, and widespread synapse and neuron loss. In addition to these lesions, oxidative stress, neuroinflammation, and aberrant cell-cycle re-entry are among the pathological features observed in AD brains (Mondragon-Rodriguez et al., 2010). The cause of $\mathrm{AD}$ is not known, and controversy persists over which abnormalities initiate the disease process, which are responses that potentiate neurodegeneration, and even whether some of these abnormalities represent protective responses of the brain (Mondragon-Rodriguez et al., 2010).

The identification of genes that influence susceptibility to $\mathrm{AD}$ has led to insights into potential disease mechanisms. Although most cases of $\mathrm{AD}$ arise sporadically, in rare cases the disease is caused by autosomal-dominant mutations. All known mutations that cause familial autosomal-dominant $\mathrm{AD}$ increase the production of $\mathrm{A} \beta$ or its propensity to aggregate (reviewed in Ashe and Zahs, 2010). Conversely, a mutation in the amyloid precursor protein (APP) that results in decreased production of $\mathrm{A} \beta$ protects against sporadic AD (Jonsson et al., 2012). The best established genetic risk factor for sporadic $\mathrm{AD}$ is the $\varepsilon 4$ allele of the $\mathrm{APOE}$ (apolipoprotein E) gene - compared to individuals with the most common genotype ( $\varepsilon 3 / \varepsilon 3)$, individuals with one copy of the $\varepsilon 4$ allele are three times more likely to develop $\mathrm{AD}$, while those with two copies of the $\varepsilon 4$ allele are 10-15 times more likely to develop
AD (Corder et al., 1993). While the exact role that APOE plays in $\mathrm{AD}$ is not known, animal studies have shown that the rate of $\mathrm{A} \beta$ clearance from the brain is differentially regulated by the various isoforms of APOE (Castellano et al., 2011). Thus, the genetic evidence points to some form of $A \beta$ - likely a pathological aggregate as the molecular trigger for $\mathrm{AD}$.

Mutations in tau, on the other hand, do not lead to AD, but to a different neurodegenerative disorder, frontotemporal dementia (FTD; Hutton et al., 1998). Transgenic mice expressing human tau with FTD-linked mutations exhibit pronounced neurodegeneration (Lewis et al., 2000; Ramsden et al., 2005; Santacruz et al., 2005; Yoshiyama et al., 2007). Notably, many of the post-translational modifications in tau that are promoted by FTD-linked mutations are also seen in AD brains (Ramsden et al., 2005; Yoshiyama et al., 2007). Furthermore, studies in animal models have shown that $\mathrm{A} \beta$ potentiates tau pathology (Gotz et al., 2001; Lewis et al., 2001; Oddo et al., 2004; Bolmont et al., 2007). Finally, tau is required for the expression of $A \beta$-induced neurological abnormalities in transgenic mice (Roberson et al., 2007).

Taken together, the evidence cited above provides strong support for the Amyloid Cascade Hypothesis, which posits that pathogenic forms of $\mathrm{A} \beta$ trigger a cascade that leads to the formation of toxic tau species and culminates in neuron death and dementia. While the Amyloid Cascade Hypothesis is arguably the primary hypothesis driving $\mathrm{AD}$ research today, it has its detractors (Castellani and Smith, 2011). Opponents of the hypothesis point to the failure in clinical trials of drugs that target amyloid; while supporters contend that treatments were administered too late to be effective - at a time when neurodegeneration had become independent of the toxic $A \beta$ species that initiated the disease process. To truly test the Amyloid Cascade Hypothesis will require 
intervening very early in the disease process, likely before symptoms are apparent, and targeting the correct form of $\mathrm{A} \beta$. The identification of the pathogenic form(s) of $A \beta$ is the focus of this Perspective.

\section{A $\beta$ OLIGOMERS IN THE HUMAN CENTRAL NERVOUS SYSTEM}

One of the most profound questions for $\mathrm{AD}$ researchers today is which $A \beta$ species triggers the amyloid cascade. In addition to amyloid plaques, which contain precipitates of fibrillar $A \beta$, several types of soluble $\mathrm{A} \beta$ assemblies ("oligomers") have been described in the brains of $\mathrm{AD}$ patients and transgenic mouse models of $\mathrm{AD}$. Studies conducted over the past decade indicate that oligomers, rather than fibrillar $\mathrm{A} \beta$, are the predominant bioactive forms; synthetic and naturally derived $A \beta$ oligomers harm cultured neurons and impair synaptic function and memory through a broad range of mechanisms (reviewed in Ashe and Zahs, 2010; Koffie et al., 2011). Identifying the oligomer that initiates the amyloid cascade is not just of academic interest - this knowledge is critical for the development of strategies to prevent AD.

Alzheimer's disease has a long preclinical phase, lasting a decade or more (Sperling et al., 2011; Bateman et al., 2012; Villemagne et al., 2013). During this preclinical phase, people appear cognitively intact (i.e., they score within the normal range on neuropsychiatric tests), but evidence of neurological disease can be seen through cerebrospinal fluid (CSF) or brain imaging analyses. It is during this preclinical phase, before the irreversible neuron loss that characterizes the dementia phase of the disease, that therapies are most likely to be effective. If we are to block $\mathrm{AD}$ during the preclinical phase, we must identify the $A \beta$ species that are present before there are overt symptoms and are associated with downstream markers of synaptic or neuronal pathology.

Two studies published this year measured levels of specific $\mathrm{A} \beta$ oligomers in the brains and CSF of human subjects - both reported that the putative dodecamer $A \beta^{\star 56}$ (Lesné et al., 2006) correlated with markers of neuronal dysfunction or injury in cognitively normal subjects. Lesné et al. (2013) measured brain $A \beta$ dimers, $A \beta$ trimers, and $A \beta \star 56$ in 140 autopsy specimens from subjects spanning 10 decades of age. In subjects who were cognitively normal at the time of death, $A \beta^{\star} 56$, but not other $A \beta$ oligomers, correlated negatively with the post-synaptic markers drebrin and Fyn kinase and positively with pathological conformers of tau (Lesné et al., 2013). In the first study to measure levels of specific $A \beta$ oligomers in the CSF of cognitively normal older adults, Handoko etal. (2013) found that $A \beta \star 56$ was elevated in individuals at risk for developing $\mathrm{AD}$ and correlated strongly with levels of total tau and tau phosphorylated at threonine-181, putative markers of neuronal injury. Interestingly, the levels of $A \beta^{\star} 56$ in the brain rose significantly in late middle age (Lesné et al., 2013) - considering the long duration of the preclinical phase of $\mathrm{AD}$, this is the time when one would expect the emergence of species that trigger the amyloid cascade.

It should be noted that other $A \beta$ assemblies, in addition to those studied by Lesné et al. (2013) and Handoko et al. (2013), have been described in the brains of AD patients (Noguchi et al., 2009; Lasagna-Reeves et al., 2011). Whether these species exist in the preclinical phase of $\mathrm{AD}$ and whether they are associated with downstream markers of pathology are yet to be determined.

\section{A $\beta$ OLIGOMERS IN TRANSGENIC MOUSE MODELS OF ALZHEIMER'S DISEASE}

Studies in animal models have provided further insights into the pathophysiological roles of specific oligomers. Among the $\mathrm{A} \beta$ species detected in the brains of $\mathrm{AD}$ patients or transgenic mouse models of $\mathrm{AD}$, only two have been shown to induce neural dysfunction when isolated and injected into the brains of healthy host animals: $A \beta \star 56$ (Lesné et al., 2006; Reed et al., 2009) and $A \beta$ dimers (Klyubin et al., 2008; Shankar et al., 2008; Barry et al., 2011; our unpublished observations). Additionally, $A \beta^{\star} 56$ correlates with memory deficits in three distinct lines of APP transgenic mice (Lesné et al., 2006, Lesné et al., 2008; Billings et al., 2007; Cheng etal., 2007). (It might at first seem inconsistent that $A \beta^{\star 56}$ is elevated in humans who are considered "cognitively intact," but that it is associated with cognitive dysfunction in rodents. However, it is very possible that cognitive decline would be revealed in these human subjects if they were tested longitudinally using sensitive neuropsychiatric instruments designed for detecting cognitive changes in clinically unimpaired individuals; Rogers, 2013.) Until recently, it has been difficult to assess the effects of $\mathrm{A} \beta$ dimers in situ (naturally located in the brain in which they were produced), due to a lack of animal models that generated dimers in the absence of $A \beta^{\star 56}$. Our laboratory recently created a novel transgenic mouse that generates abundant plaques and $A \beta$ dimers but negligible levels of other $A \beta$ oligomers. These mice remain cognitively normal, even at advanced ages. This result was surprising in view of the ex situ studies demonstrating the toxicity of $\mathrm{A} \beta$ dimers, cited above. To explain these paradoxical findings, we hypothesized that dimers in situ are compartmentalized in a way that limits their toxicity. Biochemical studies have shown an intimate relationship between dimers and plaques (Roher et al., 1996; Shankar et al., 2008). Using laser microdissection followed by immunoblotting, we found that dimers in situ are confined to the immediate vicinity of plaques, while $A \beta^{\star 56}$ is diffusely distributed throughout the brain parenchyma (Liu et al., 2011). We concluded that despite their potent neurotoxicity when dispersed into cell cultures (Roher etal., 1996; Jin et al., 2011) or into the brains of experimental hosts (Klyubin et al., 2008; Shankar et al., 2008; Barry et al., 2011), dimers in situ exert few or no large-scale effects on brain function.

This conclusion highlights the necessity of studying candidate pathogenic molecules/processes in situ in order to gain a more genuine understanding of their effects in the brain. While in vitro studies can provide valuable information about cellular mechanisms of action, cell culture conditions do not mimic the spatiotemporal expression of pathogenic molecules or the complex intercellular interactions that occur in vivo. Similarly, exogenous administration of candidate pathogenic molecules can show the toxic potential of these molecules, but not necessarily their effects on neurological function when naturally produced and localized in the brain. This caution does not apply only to studies of $A \beta$ - a recent essay in this series argued that extrapolating the results of in vitro studies to the situation in vivo has resulted in 
a gross misunderstanding of the role of microglia in chronic brain disease (Streit, 2010).

\section{DUAL-PATHWAY MODEL FOR A $\beta$ TOXICITY IN THE BRAIN}

Based on the findings discussed above, we suggest that there are at least two ways in which $A \beta$ oligomers can be toxic, as illustrated in the model in Figure 1. In this model, $A \beta^{\star} 56$ is diffusely distributed throughout the brain parenchyma and induces widespread synaptic dysfunction, which eventually leads to pathological processing of tau and subsequent neurodegeneration over a wide area. $A \beta$ dimers are potently neurotoxic and induce neurodegeneration, but the tight sequestration of dimers to the immediate vicinity of plaques spatially restricts their influence. This model has important implications for the development of strategies to prevent $\mathrm{AD}$. We hypothesize that, in order to prevent or delay the onset of dementia, anti-amyloid therapies must not only be administered early in the presymptomatic phase of the disease, but must target $A \beta^{\star} 56$. According to the model, strategies that target $\mathrm{A} \beta$ dimers and and/or fibrils might correct plaque-associated neurodegenerative changes if administered early enough, but will have little impact on the development of the widespread neuronal dysfunction and degeneration that characterize AD.

\section{THE ROLE OF A $\beta$ IN AGE-RELATED COGNITIVE DECLINE}

As mentioned in the Introduction, age is the greatest risk factor for $\mathrm{AD}$. It is not known whether $\mathrm{AD}$ falls at the extreme end of a continuum of normal age-related cognitive decline or whether it represents a distinct pathological process. The same mutation in APP that protects against AD also protects against age-related cognitive decline (Jonsson et al., 2012), suggesting that $\mathrm{A} \beta$ has a role in both conditions. Our laboratory previously reported that expression of transgenic APP accelerated the appearance of age-related neural dysfunction in mice (Hsiao etal., 1995). These studies occurred prior to the time that we began characterizing soluble oligomers in the brains of our mice, so we do not know which $A \beta$ species were involved, although notably these mice did not generate plaques. Observations in senescence-accelerated prone mice (SAMP) are consistent with the hypothesis that $A \beta$ is involved in "normal" brain aging; these mice develop deficits in learning and memory at young ages relative to the parent strain and have elevated levels of endogenous mouse APP and A $\beta$, but no plaques (Okuma and Nomura, 1998). Administration of antibodies against $A \beta$ or anti-sense nucleotides that lower APP mRNA ameliorates cognitive deficits in these mice (Kumar et al., 2000; Morley et al., 2000; Banks et al., 2001).

\section{FUTURE DIRECTIONS AND CONCLUSIONS}

The data available thus far strongly suggest that $A \beta \star 56$ is critically involved in the earliest stages of AD. However, longitudinal studies are required to determine whether "cognitively normal" individuals with elevated levels of $\mathrm{A} \beta \star 56$ show accelerated rates of cognitive decline or are indeed more likely to develop $\mathrm{AD}$ than people with low levels of $A \beta^{\star} 56$. These studies might also shed light on the question of whether age-related cognitive decline and AD share similar mechanisms and are indeed on a continuum of brain aging. Such studies are still only correlative. To really test the hypothesis that $A \beta^{\star} 56$ is necessary to trigger the amyloid cascade, we must determine whether selectively decreasing the levels of $A \beta^{\star} 56$, or interfering with its interactions with its

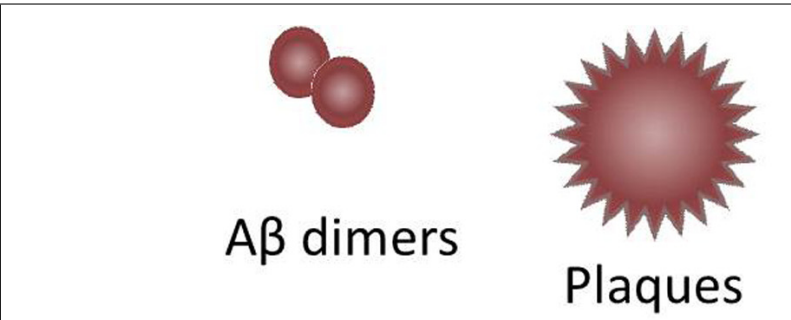

\section{Neurodegeneration \\ $\rightarrow$ (highly restricted) Normal cognition}

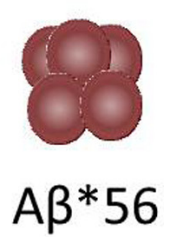

FIGURE 1 | Dual-pathway model for $A \boldsymbol{\beta}$ toxicity in the brain. The model illustrates two ways in which $A \beta$ oligomers can be toxic: (1) $A \beta^{*} 56$, which is diffusely distributed throughout the brain parenchyma, induces widespread synaptic dysfunction, leading to pathological processing of tau and subsequent neurodegeneration over a wide area.

\section{Normal neuronal morphology Impaired cognition}

(2) A $\beta$ dimers, which are tightly sequestered around plaques, induce neurodegeneration only in the immediate vicinity of plaques. The area influenced by dimers is not large enough to impair cognitive function in animals with plaque loads that approximate those found in humans. 
cellular targets, reduces the risk of symptomatic AD. Such studies await the development of reagents that selectively target specific $A \beta$ oligomers.

Three well publicized $\mathrm{AD}$ prevention trials are scheduled to begin this year (2013): the Alzheimer's Prevention Initiative (API) and Dominantly Inherited Alzheimer's Network (DIAN) trials will test anti-amyloid therapies in asymptomatic members of families with autosomal-dominant AD, while the Anti-amyloid Treatment in Asymptomatic Alzheimer's Disease (A4) trial will examine the effects of therapy on individuals at increased risk of sporadic $\mathrm{AD}$, enrolling elderly people with who have amyloid-positive PET scans. All of these trials will test the effects of monoclonal antibodies directed against $A \beta$, but it is not known whether any of

\section{REFERENCES}

Alzheimer's Association. (2012). 2012 Alzheimer's disease facts and figures. Alzheimers Dement. 8, 131-168. doi: 10.1016/j.jalz.2012.02.001

Ashe, K. H., and Zahs, K. R. (2010). Probing the biology of Alzheimer's disease in mice. Neuron 66, 631-645. doi: 10.1016/j.neuron.2010.04.031

Banks, W. A., Farr, S. A., Butt, W., Kumar, V. B., Franko, M. W., and Morley, J. E. (2001). Delivery across the blood-brain barrier of antisense directed against amyloid beta: reversal of learning and memory deficits in mice overexpressing amyloid precursor protein. J. Pharmacol. Exp. Ther. 297, 1113-1121.

Barry, A. E., Klyubin, I., Mc Donald, J. M., Mably, A. J., Farrell, M. A., Scott, M., et al. (2011). Alzheimer's disease brainderived amyloid-beta-mediated inhibition of LTP in vivo is prevented by immunotargeting cellular prion protein. J. Neurosci. 31, 72597263. doi: 10.1523/JNEUROSCI. 6500-10.2011

Bateman, R. J., Xiong, C., Benzinger, T. L., Fagan, A. M., Goate, A., Fox, N. C., etal. (2012). Clinical and biomarker changes in dominantly inherited Alzheimer's disease. N. Engl. J. Med. 367, 795-804. doi: 10.1056/NEJMoa1202753

Billings, L. M., Green, K. N., Mcgaugh, J. L., and Laferla, F. M. (2007). Learning decreases A beta $^{\star} 56$ and tau pathology and ameliorates behavioral decline in $3 \times \mathrm{Tg}$ AD mice. J. Neurosci. 27, 751-761. doi: 10.1523/JNEUROSCI.4800-06. 2007

Bolmont, T., Clavaguera, F., MeyerLuehmann, M., Herzig, M. C., Radde, R., Staufenbiel, M., et al. (2007). Induction of tau pathology by intracerebral infusion of amyloidbeta-containing brain extract and by amyloid-beta deposition in $\mathrm{APP} \times$ Tau transgenic mice. Am.
J. Pathol. 171, 2012-2020. doi: 10.2353/ajpath.2007.070403

Castellani, R. J., and Smith, M. A. (2011). Compounding artefacts with uncertainty, and an amyloid cascade hypothesis that is 'too big to fail'. J. Pathol. 224, 147-152. doi: 10.1002/path.2885

Castellano, J. M., Kim, J., Stewart, F. R., Jiang, H., Demattos, R. B., Patterson, B. W., et al. (2011). Human apoE isoforms differentially regulate brain amyloid- $\beta$ peptide clearance. Sci. Transl. Med. 3, 89ra57. doi: 10.1126/scitranslmed.3002156

Cheng, I. H., Scearce-Levie, K., Legleiter, J., Palop, J. J., Gerstein, H., Bien-Ly, N., etal. (2007). Accelerating amyloid-beta fibrillization reduces oligomer levels and functional deficits in Alzheimer disease mouse models. J. Biol. Chem. 282, 23818-23828. doi: 10.1074/ jbc.M701078200

Corder, E. H., Saunders, A. M., Strittmatter, W. J., Schmechel, D. E., Gaskell, P. C., Small, G. W., et al. (1993). Gene dose of apolipoprotein E type 4 allele and the risk of Alzheimer's disease in late onset families. Science 261, 921-923. doi: 10.1126/science. 8346443

Gotz, J., Chen, F., Van Dorpe, J., and Nitsch, R. M. (2001). Formation of neurofibrillary tangles in P301l tau transgenic mice induced by Abeta 42 fibrils. Science 293, 1491-1495. doi: $10.1126 /$ science. 1062097

Handoko, M., Grant, M., Kuskowski, M., Zahs, K. R., Wallin, A., Blennow K., et al. (2013). Correlation of specific amyloid- $\beta$ oligomers with Tau in cerebrospinal fluid from cognitively normal older adults. JAMA Neurol. 1-6. doi: 10.1001/jamaneurol.2013.48

Hsiao, K. K., Borchelt, D. R., Olson, K., Johannsdottir, R., Kitt, C., Yunis, W., etal. (1995). Agerelated CNS disorder and early death in transgenic $\mathrm{FVB} / \mathrm{N}$ mice

the antibodies to be tested in these critical prevention trials target $\mathrm{A} \beta \beta^{\star} 56$. The API, DIAN, and A4 trials are considered by many to be the first true tests of the Amyloid Cascade Hypothesis. Should these trials fail because they did not target the relevant $A \beta$ species, the result could be the premature rejection of the amyloid cascade hypothesis and the abandonment of drug development programs that target $A \beta$. Continued failures of clinical trials will further undermine public confidence in biomedical research and, most importantly, delay the implementation of therapies that will lessen the impending public health crisis that is $\mathrm{AD}$. Future research clarifying which forms of $\mathrm{A} \beta$ to target and where the targets are located will be critical for the development of mechanism-based therapies for AD.

overexpressing Alzheimer amyloid precursor proteins. Neuron 15, 1203 1218. doi: 10.1016/0896-6273(95) 90107-8

Hutton, M., Lendon, C. L., Rizzu, P., Baker, M., Froelich, S., Houlden, H., et al. (1998). Association of missense and $5^{\prime}$-splice-site mutations in tau with the inherited dementia FTDP-17. Nature 393, 702-705. doi: 10.1038/31508

Jin, M., Shepardson, N., Yang, T., Chen, G., Walsh, D., and Selkoe, D. J. (2011). Soluble amyloid beta-protein dimers isolated from Alzheimer cortex directly induce Tau hyperphosphorylation and neuritic degeneration. Proc. Natl. Acad. Sci. U.S.A. 108, 5819-5824. doi: 10.1073/pnas. 1017033108

Jonsson, T., Atwal, J. K., Steinberg, S., Snaedal, J., Jonsson, P. V., Bjornsson, S., et al. (2012). A mutation in APP protects against Alzheimer's disease and age-related cognitive decline. Nature 488, 96-99. doi: 10.1038/nature 11283

Klyubin, I., Betts, V., Welzel, A. T., Blennow, K., Zetterberg, H., Wallin, A., et al. (2008). Amyloid beta protein dimer-containing human CSF disrupts synaptic plasticity: prevention by systemic passive immunization. J. Neurosci. 28, 42314237. doi: 10.1523/JNEUROSCI. 5161-07.2008

Koffie, R. M., Hyman, B. T., and SpiresJones, T. L. (2011). Alzheimer's disease: synapses gone cold. Mol. Neurodegener. 6, 63. doi: 10.1186/ 1750-1326-6-63

Kumar, V. B., Farr, S. A., Flood, J. F., Kamlesh, V., Franko, M., Banks, W. A., et al. (2000). Site-directed antisense oligonucleotide decreases the expression of amyloid precursor protein and reverses deficits in learning and memory in aged SAMP8 mice. Peptides 21, 1769 1775. doi: 10.1016/S0196-9781(00) 00339-9
Lasagna-Reeves, C. A., Glabe, C. G., and Kayed, R. (2011). Amyloid-beta annular protofibrils evade fibrillar fate in Alzheimer disease brain. J. Biol. Chem. 286, 22122-22130. doi: 10.1074/jbc.M111.236257

Lesné, S., Koh, M. T., Kotilinek, L., Kayed, R., Glabe, C. G., Yang, A., et al. (2006). A specific amyloid-beta protein assembly in the brain impairs memory. Nature 440, 352-357. doi: 10.1038 /nature 04533

Lesné, S., Kotilinek, L., and Ashe, K. H. (2008). Plaque-bearing mice with reduced levels of oligomeric amyloid-beta assemblies have intact memory function. Neuroscience 151, 745-749. doi: 10.1016/ j.neuroscience.2007.10.054

Lesné, S. E., Sherman, M., Grant, M., Kuskowski, M., Schneider, J. A., Bennett, D. A., et al. (2013). Brain amyloid- $\beta$ oligomers in aging and Alzheimer's disease. Brain 136(Pt 5), 1383-1398. doi: 10.1093/brain/awt062

Lewis, J., Dickson, D. W., Lin, W. L., Chisholm, L., Corral, A., Jones, G., etal. (2001). Enhanced neurofibrillary degeneration in transgenic mice expressing mutant tau and APP. Science 293, 1487-1491. doi: 10.1126/science.1058189

Lewis, J., Mcgowan, E., Rockwood, J., Melrose, H., Nacharaju, P., Van Slegtenhorst, M., et al. (2000). Neurofibrillary tangles, amyotrophy and progressive motor disturbance in mice expressing mutant $(\mathrm{P} 301 \mathrm{~L})$ tau protein. Nat. Genet. 25, 402-405. doi: $10.1038 / 78078$

Liu, P., Forster, C., Kotilinek, L., Paulson, J., Chen, G., Bennett, D., et al. (2011). Abeta dimers mediate plaque-associated cytopathology without affecting cognition. Alzheimers Dement. 7, e23. doi: 10.1016/j.jalz.2011.09.067

Mondragon-Rodriguez, S., BasurtoIslas, G., Lee, H. G., Perry, G., Zhu, X., Castellani, R. J., et al. (2010). 
Causes versus effects: the increasing complexities of Alzheimer's disease pathogenesis. Expert Rev. Neurother. 10, 683-691. doi: 10.1586/ ern.10.27

Morley, J. E., Kumar, V. B., Bernardo, A. E., Farr, S. A., Uezu, K., Tumosa, N., et al. (2000). Beta-amyloid precursor polypeptide in SAMP8 mice affects learning and memory. Peptides 21, 1761-1767. doi: 10.1016/S01969781(00)00342-9

Noguchi, A., Matsumura, S., Dezawa, M., Tada, M., Yanazawa, M., Ito A., et al. (2009). Isolation and characterization of patient-derived, toxic, high mass amyloid beta-protein (Abeta) assembly from Alzheimer disease brains. J. Biol. Chem. 284, 32895-32905. doi: 10.1074/ jbc.M109.000208

Oddo, S., Billings, L., Kesslak, J. P., Cribbs, D. H., and Laferla, F. M. (2004). Abeta immunotherapy leads to clearance of early, but not late, hyperphosphorylated tau aggregates via the proteasome. Neuron 43 321-332. doi: 10.1016/j.neuron.2004. 07.003

Okuma, Y., and Nomura, Y. (1998). Senescence-accelerated mouse (SAM) as an animal model of senile dementia: pharmacological, neurochemical and molecular biological approach. Jpn. J. Pharmacol. 78, 399-404. doi: 10.1254/jjp.78.399

Ramsden, M., Kotilinek, L., Forster, C., Paulson, J., Mcgowan, E., Santacruz,
K., et al. (2005). Age-dependent neurofibrillary tangle formation, neuron loss, and memory impairment in a mouse model of human tauopathy (P301L). J. Neurosci. 25, 10637-10647. doi: 10.1523/JNEUROSCI.3279-05.2005

Reed, M. N., Hofmeister, J. J., Jungbauer, L., Welzel, A. T., Yu, C., Sherman, M. A., et al. (2009). Cognitive effects of cell-derived and synthetically derived Abeta oligomers. Neurobiol. Aging 32, 1784-1794. doi: 10.1016/j.neurobiolaging.2009. 11.007

Roberson, E. D., Scearce-Levie, K., Palop, J. J., Yan, F., Cheng, I. H., Wu, T., et al. (2007). Reducing endogenous tau ameliorates amyloid beta-induced deficits in an Alzheimer's disease mouse model. Science 316, 750-754. doi: 10.1126/science. 1141736

Rogers, M. B. (2013). New Frontier: Developing Outcome Measures for Predementia Trials [online]. Available at: http://www.alzforum.org/res/for/ journal/detail.asp?liveID $=208$ [accessed March 18, 2013].

Roher, A. E., Chaney, M. O., Kuo, Y. M., Webster, S. D., Stine, W. B., Haverkamp, L. J., et al. (1996). Morphology and toxicity of Abeta-(1-42) dimer derived from neuritic and vascular amyloid deposits of Alzheimer's disease. J. Biol. Chem. 271, 2063120635. doi: 10.1074/jbc.271.34 2063
Santacruz, K., Lewis, J., Spires, T., Paulson, J., Kotilinek, L., Ingelsson, M., et al. (2005). Tau suppression in a neurodegenerative mouse model improves memory function. Sci ence 309, 476-481. doi: 10.1126/science. 1113694

Shankar, G. M., Li, S., Mehta, T. H., Garcia-Munoz, A., Shepardson, N. E. Smith, I., et al. (2008). Amyloid-beta protein dimers isolated directly from Alzheimer's brains impair synaptic plasticity and memory. Nat. Med. 14, 837-842. doi: 10.1038/ $\mathrm{nm} 1782$

Sperling, R. A., Aisen, P. S., Beckett, L. A., Bennett, D. A., Craft, S., Fagan, A. M., et al. (2011). Toward defining the preclinical stages of Alzheimer's disease: recommendations from the National Institute on Aging-Alzheimer's Association workgroups on diagnostic guidelines for Alzheimer's disease. Alzheimers Dement. 7, 280-292. doi: 10.1016/j.jalz.2011.03.003

Streit, W. J. (2010). Microglial activation and neuroinflammation in Alzheimer's disease: a critical examination of recent history. Front. Aging Neurosci. 2:22. doi: 10.3389/fnagi.2010.00022

Villemagne, V. L., Burnham, S. Bourgeat, P., Brown, B., Ellis, K. A., Salvado, O., et al. (2013). Amyloid beta deposition, neurodegeneration, and cognitive decline in sporadic Alzheimer's disease: a prospective cohort study. Lancet Neurol. 12, 357-367. doi: 10.1016/S14744422(13)70044-9

Yoshiyama, Y., Higuchi, M., Zhang, B., Huang, S. M., Iwata, N., Saido, T. C., et al. (2007). Synapse loss and microglial activation precede tangles in a P301S tauopathy mouse model. Neuron 53, 337-351. doi: 10.1016/j.neuron.2007.01.010

Conflict of Interest Statement: The authors declare that the research was conducted in the absence of any commercial or financial relationships that could be construed as a potential conflict of interest.

Received: 25 March 2013; accepted: 17 June 2013; published online: 04 July 2013.

Citation: Zahs KR and Ashe KH (2013) $\beta$-Amyloid oligomers in aging and Alzheimer's disease. Front. Aging $\mathrm{Neu}$ rosci. 5:28. doi: 10.3389/fnagi.2013. 00028

Copyright (c) 2013 Zahs and Ashe. This is an open-access article distributed under the terms of the Creative Commons Attribution License, which permits use, distribution and reproduction in other forums, provided the original authors and source are credited and subject to any copyright notices concerning any thirdparty graphics etc. 